\title{
Novel Therapeutics Targeting Telomerase and Telomeres
}

Neelu Puri* and Jennifer Girard

Department of Biomedical Sciences, University of Illinois at Chicago, USA

\section{Introduction}

Telomerase is a ribonucleoprotein enzyme that binds to the telomeres, increases their length, and extends the lifespan of cells. Telomerase is expressed at low or undetectable levels in normal somatic tissue. However, telomerase is expressed at detectable levels in a wide range of cancer cells, including brain, skin, lung and blood. The selective high level of expression of telomerase in cancer cells makes it an attractive target for cancer therapy. Researchers have investigated cancer therapeutics targeting telomerase and its mechanism of action by multiple approaches, including vaccines, small molecule inhibitors, oligonucleotides and G-quadruplex approaches. Several therapeutics targeting telomerase, such as Imetelstat (GRN163L) and Tertomotide (GV1001), are currently in Phase I, II and III clinic trials to treat a wide range of cancers from pediatric solid tumors to non-small cell lung cancer, leukemia, lymphoma and myeloma.

Therapeutics inhibiting telomerase work by a variety of mechanisms. These mechanisms include direct inhibition of telomerase, generation of immune response by vaccines, stabilization of G-quadruplexes and induction of DNA damage responses by T-oligo. There are two well-studied direct inhibitors of telomerase, GRN163L and BIBR1532. The GRN163L, a 13-mer oligonucleotide, inhibits telomerase directly by antagonistic binding with high affinity to the RNA template of telomerase (hTERC) [1]. Imetelstat is currently in a number of clinical trials, as described below. BIBR1532 acts as a non-competitive nonnucleoside inhibitor of telomerase, impairing substrate elongation by shortening TTAGGG repeats [2]. BIBR1532 has had preclinical success by inhibiting cell growth without significant cytotoxicity and sensitizing drug-resistant cell lines to alterative chemotherapies $[3,4]$, however, these studies have not progressed into clinical trials. Another class of therapeutics, the cancer vaccines, includes GV1001, Vx-001 and GRNVAC1/GRNVAC2. GV1001 is a peptide vaccine adjuvanted with GM-CSF that produces a robust CD4+ cell response [5] and is currently in Phase III clinical trials, which is further discussed below. The cryptic peptide based vaccine $\mathrm{Vx}-001$ showed success in Phase I/II clinical trials by meeting primary outcomes of safety and tolerability, and by generating a strong immune response in $70 \%$ of patients [6]. Vaxon-Biotech (Paris, France) has also initiated their randomized multicenter Phase IIb trial with $\mathrm{Vx}-001$ for the treatment of non-small-cell lung cancer in 2012 [7]. GRNVAC1/GRNVAC2 dendritic cell based vaccines have also met the safety and tolerability primary outcomes of a Phase I clinical trial. A Phase II clinical trial for the treatment of acute myelogenous leukemia is currently ongoing, with a targeted completion of 2015 [8]. Other telomerase inhibitors, such as telomestatin, BRACO-19 and RHPS4, stabilize and form the G-quadruplex (GGTTAG) structure, thereby decreasing the efficiency of telomerase $[9,10]$.

T-oligo, an 11-base oligonucleotide sequence (GTTAGGGTTAG), does not directly target telomerase, but instead mimics the exposure of the 3 single strand telomeric sequence overhang. T-oligo thereby induces DNA damage responses by activation of ATM, p53, E2F1 and p95/NBS1, thus leading to apoptosis of cancer cells in a telomerase independent manner [11-13]. Another novel approach thought to inhibit telomerase is the targeting of tankyrase 1 , a telomere specific poly (ADP-ribosyl) polymerase (PARP). Inhibition of tankyrase leads to telomere shortening through lack of ribosylation of telomere repeat binding factor 1 (TRF1) protein, an integral component of the shelterin complex. Tankyrase inhibitors, XAV939 and 3-aminobenzamide $(3 \mathrm{AB})$, have been shown to initiate downstream DNA damage responses [14].

\section{Clincal Trials}

One of the most promising therapeutics that targets telomerase is GRN163L. GRN163L is a 13- mer oligonucleotide with a thiophophoramidate backbone, which makes it resistant to nucleases with lipophilic palmitoyl thio phosphate, allowing for increased intracellular uptake by targeting hTERC [15]. GRN163L is involved in 14 clinical trials, including four Phase II and ten Phase I clinical trials, which are targeting pediatric solid tumors, non small cell lung cancer (NSCLC), breast cancer and blood ascariases. The clinical trials involving the treatment of pediatric solid tumors are led by the National Cancer Institute, while trials targeting adult tumors are sponsored by Geron. In 2013, the Pediatric Brain Tumor Consortium, in collaboration with the National Cancer Institute, initiated the recruitment of pediatric patients with recurrent High-Grade Glioma, Ependymoma, and Medulloblastoma to test the efficacy of GRN163L. The main outcome of this trial determined the percentage of subjects with telomerase-positive tumors demonstrating greater than 50\% reduction in telomerase activity [16].

The remaining eight Phase I and three Phase II clinical trials targeting adult cancers are sponsored by Geron Corporation. GRN163L met its primary outcomes, including safety, tolerability and identification of maximum tolerable dose, by using intermittent dosing due to major toxicities of thrombocytopenia and neutropenia. Based on these results, Phase II trials for the treatment of NSCLC, thrombocythemia and metastatic breast cancer with combination treatments have been initiated. Preliminary data from the Phase II trial targeting NSCLC presented at the 2013 AACR demonstrated no clinically significant trend toward Progression Free Survival (PFS), with a trend toward improved Overall Survival (OS) that was not statistically significant [17]. Interestingly, in a subset of the patients in this trial whose tumors had short telomeres, there was a trend towards PFS and OS that suggested a survival benefit [18]. Results from the Phase II multiple-center clinical trial of GRN163L for the treatment of essential thrombocythemia were presented at the 2013 Congress of the

*Corresponding author: Neelu Puri Ph.D. Assistant Professor, Department of Biomedical Sciences, University of Illinois at Chicago, Rockford College of Medicine Campus, Room Number E-632, 1601 Parkview Ave, Rockford, II-61107, USA, Tel: 815395 5678; E-mail: neelupur@uic.edu

Received September 18, 2013; Accepted September 20, 2013; Published September 23, 2013

Citation: Puri N, Girard J (2013) Novel Therapeutics Targeting Telomerase and Telomeres. J Cancer Sci Ther 5: e127. doi:10.4172/1948-5956.1000e127

Copyright: @ 2013 Puri N, et al. This is an open-access article distributed under the terms of the Creative Commons Attribution License, which permits unrestricted use, distribution, and reproduction in any medium, provided the original author and source are credited. 
European Hematology Association, which included 18 patients with essential thrombocythemia that were refractory to initial treatment. Significantly, complete response was demonstrated in $16 / 18$ patients at a rate of $89 \%$. Among these 16 patients, eight patients had the JAK2 V617F mutation and demonstrated a partial response rate of $86 \%$ in decreasing the allele load [19]. The last of the Phase II trials sponsored by Geron involved GRN163L therapy in combination with paclitaxel with or without bevacizumab. This approach established that treatment of breast cancer stem cells with GRN163L in combination with a tumor debulking agent, such as paclitaxel, could lead to a more robust therapeutic response. Phase I trials of GRN163L in combination with paclitaxel and bevacizumab in patients with local recurrent or metastatic breast cancer demonstrated an objective response rate of $54.8 \%(28.7-77.6 \%)$. However, $78.6 \%$ of patients required reduced doses of Paclitaxel and/or GRN163L due to thrombocytopenia and neutropenia, with responses in five out of the 14 patients [20]. The primary endpoint for the future Phase II trial is PFS with secondary efficacy endpoints, including response rate and clinical benefit. This trial estimates enrollment of 150 patients at 80 clinical sites in the United States and Canada.

GV1001 is a peptide vaccine consisting of a 15 amino acid epitope of hTERT, the enzymatic component of telomerase. Sargramostim (GM-CSF) is administered simultaneously to stimulate immune response. Vaccination with the GV1001 peptide leads to $\mathrm{CD}^{+} \mathrm{T}$ cells that recognize hTERT [5]. Most recently, GV1001 was added to standard gemcitabine/capeciabine for the treatment of locally advanced or metastatic pancreatic cancer in a Phase III trial (TeloVac trial) that involved a 1,062 patient, and 51 center study. Results presented at the 2013 AACR concluded no survival benefit over the standard gemcitabine/capecibine treatment [21]. However, there is still promise for GV1001 as a treatment for NSCLC. A Phase II trial involving 38 patients with NSCLC demonstrated an immune response in 54\% of patients after four weeks of treatment and in $86 \%$ of patients after eight weeks of treatment, without serious side effects [22]. KAEL-GemVax is sponsoring a Phase III clinical trial for the treatment of NSCLC that is approved but not yet active.

\section{Conclusion}

A number of telomerase inhibitors are involved in large Phase II and III clinical trials with moderate successes, albeit not without failures along the way. GRN163L has demonstrated little or no benefit to PFS and OS in NSCLC, according to a Phase II clinical trial. The most promising use of GRN163L as a cancer therapeutic is in the treatment of essential thrombocythemia. Additionally, GV1001 vaccine did not show any survival benefit in a Phase III clinical trial for the treatment of pancreatic adenocarcinoma, but a Phase II clinical trial targeting NSCLC shows promise with an immune response rate in greater than $50 \%$ of the patients. There are still a number of telomerase inhibitor candidates in the pipeline that have yet to make it to clinical trials. T-oligo shows promise in preclinical studies, however, the major hurdle to starting clinical trials is the development of a non-toxic delivery system to increase intracellular uptake of T-oligo in vivo. Currently, the most novel approach to the inhibition of telomerase activity in cancer cells relies on the inhibition of tankyrase-1, which induces shortening of telomeres in cancer cells and initiates DNA damage responses. Further studies will determine the therapeutic efficacy of these various anticancer agents targeting telomerase, which are currently in various stages of development.

\section{References}

1. Röth A, Harley CB, Baerlocher GM (2010) Imetelstat (GRN163L)--telomerasebased cancer therapy. Recent Results Cancer Res 184: 221-234.

2. Pascolo E, Wenz C, Lingner J, Hauel N, Priepke H, et al. (2002) Mechanism of human telomerase inhibition by BIBR1532, a synthetic, non-nucleosidic drug candidate. J Biol Chem 277: 15566-15572.

3. El-Daly H, Kull M, Zimmermann S, Pantic M, Waller CF, et al. (2005) Selective cytotoxicity and telomere damage in leukemia cells using the telomerase inhibitor BIBR1532. Blood 105: 1742-1749.

4. Ward RJ, Autexier C (2005) Pharmacological telomerase inhibition can sensitize drug-resistant and drug-sensitive cells to chemotherapeutic treatment. Mo Pharmacol 68: 779-786.

5. Inderberg-Suso EM, Trachsel S, Lislerud K, Rasmussen AM, Gaudernack G (2012) Widespread CD4+ T-cell reactivity to novel hTERT epitopes following vaccination of cancer patients with a single hTERT peptide GV1001. Oncoimmunology 1: 670-686.

6. Kotsakis A, Vetsika EK, Christou S, Hatzidaki D, Vardakis N, et al. (2012) Clinical outcome of patients with various advanced cancer types vaccinated with an optimized cryptic human telomerase reverse transcriptase (TERT) peptide: results of an expanded phase II study. Ann Oncol 23: 442-449.

7. Georgoulias V, Douillard JY, Khayat D, Manegold C, Rosell R, et al. (2013) A multicenter randomized phase IIb efficacy study of $\mathrm{Vx}-001$, a peptide-based cancer vaccine as maintenance treatment in advanced non-small-cell lung cancer: treatment rationale and protocol dynamics. Clin Lung Cancer 14: 461465

8. DiPersio J (2007) A Study of Active Immunotherapy With GRNVAC1 in Patients With Acute Myelogenous Leukemia (AML).

9. Harley CB (2008) Telomerase and cancer therapeutics. Nat Rev Cancer 8 167-179.

10. $\mathrm{Xu} Y$ (2011) Chemistry in human telomere biology: structure, function and targeting of telomere DNA/RNA. Chem Soc Rev 40: 2719-2740.

11. Yaar M, Eller MS, Panova I, Kubera J, Wee LH, et al. (2007) Telomeric DNA induces apoptosis and senescence of human breast carcinoma cells. Breast Cancer Res 9: R13.

12. Eller MS, Puri N, Hadshiew IM, Venna SS, Gilchrest BA (2002) Induction of apoptosis by telomere 3' overhang-specific DNA. Exp Cell Res 276: 185-193.

13. Longe HO, Romesser PB, Rankin AM, Faller DV, Eller MS, et al. (2009) Telomere homolog oligonucleotides induce apoptosis in malignant but not in normal lymphoid cells: mechanism and therapeutic potential. Int J Cancer 124: 473-482.

14. Pitman RT, Wojdyla L, Puri N (2013) Mechanism of DNA damage responses induced by exposure to an oligonucleotide homologous to the telomere overhang in melanoma. Oncotarget 4: 761-771.

15. Ruden M, Puri N (2013) Novel anticancer therapeutics targeting telomerase Cancer Treat Rev 39: 444-456.

16. Fouladi M (2013) Imetelstat Sodium in Treating Younger Patients With Recurrent or Refractory Brain Tumors.

17. Chiappori A, Kolevska T, Burington B, Spiegel DR, Hager S, et al. (2013) A randomized Phase II STudy of the Telomerase Inhibitor Imetelstat as a Maintenance Therapy for Advanced Non-Small Cell Lung Cancer. Annual Meeting 2013, Washington, DC

18. Chiappori A, Bassett K Burington B, Spiegel DR, Hager S et al. (2013) Improved Progression Free Survival (PFS) in Patients with Short Tumor Telomere Length: Subgroup Analysis from a Randomized Phase II Study of the Telomerase Inhibitor Imetelstat as a Maintenance Therapy for Advanced NSCLC.

19. Baerlocher G, Oppliger Leibundgut E, Spitzer G, Ottmann O, Olatoyosi O, et al (2013) Imetelstat: A Novel Approach with Robust Hematologic and Molecular Responses in a Phase II Study in Patients with Essential Thrombocythemia Who are Refractory or Intolerant to Prior Therapy. 2013 Congress of the European Hematology Association

20. M Kozloff GWS, FM Benedetti, A Starr, JA Wallace, MJ Stuart, et al. (2010) Phase I study of imetelstat (GRN163L) in combination with paclitaxel (P) and bevacizumab (B) in patients (pts) with locally recurrent or metastatic breast cancer (MBC). J Clin Oncol 28: 15s. 
Citation: Puri N, Girard J (2013) Novel Therapeutics Targeting Telomerase and Telomeres. J Cancer Sci Ther 5: e127. doi:10.4172/1948$5956.1000 \mathrm{e} 127$

Page 3 of 3

21. Middleton G, Valle J, Wadsley J, Propper D, Coxon F, et al. (2013) A phase III randomized trial of chemoimmunotherapy comprising gemcitabine and capecitabine with or without telomerase vaccine GV1001 in patients with locally advanced or metastatic pancreatic cancer. J Clin Oncol (suppl; abstr LBA4004).
22. Brunsvig PF, Aamdal S, Gjertsen MK, Kvalheim G, Markowski-Grimsrud CJ, et al. (2006) Telomerase peptide vaccination: a phase I//I study in patients with non-small cell lung cancer. Cancer Immunol Immunother 55: 1553-1564. 